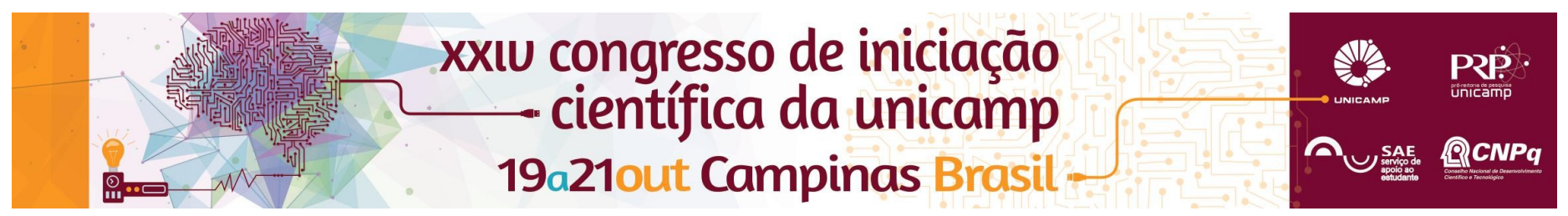

\title{
Validation of a small scale and a low cost anaerobic system for microbial applications
}

\section{Laura F. A. Meirelles*, José Valdo Madeira Jr., Vijayendran Raghavendran, Andreas K. Gombert}

\begin{abstract}
Many of the industrial building block chemicals are produced using anaerobic fermentations. Understanding the physiology of such microorganisms is vital for metabolic engineering. However, achieving fully anaerobic conditions in the laboratory is quite difficult because of the high surface area to volume ratio involved [2]. To overcome the issue of cost (anaerobic chambers have high operating cost) and to facilitate a certain degree of through-put, we developed a low cost anaerobic set-up. The aim of this project is to validate a system by cultivating different yeast strains (Kluyveromyces lactis and Saccharomyces cerevisiae). Therefore, the development of this system will allow researches on ethanol production and many other anaerobic processes to ensure the absence of oxygen in their experiments.
\end{abstract}

\section{Key words:}

Anaerobic set up, diffusion, oxygen.

\section{Introduction}

Considering the importance that biochemical processes have achieved over the years and also the high value-added compounds obtained through anaerobic reactions, having a system that can provide an anaerobic condition is of utmost importance.

Obtaining a fully anaerobic condition in a large scale process does not create any difficulty, nevertheless, achieving the absence of oxygen in a laboratory scale is extremely hard [2].

What we know:

- Kluyveromyces lactis is reported as a strict aerobic yeast (negative control) [1];

- Saccharomyces cerevisiae is able to grow in the presence or absence of oxygen (positive control) [1];

- The experiments in synthetic medium will allow us to ensure that the growth observed in the previous experiments with complex medium does not have any relationship with the presence of oxygen;

\section{Results and Discussion}

The methodology used:

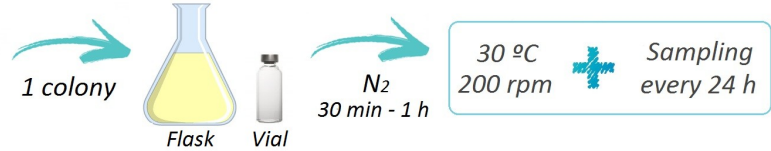

Image 1. Methodology of the experiment

The absorbance and the glucose percentage were measured for every sample and a growth curve was obtained.

In the absence of oxygen and without the cofactors oleate and ergosterol, Kluyveromyces lactis should not grow. Despite that, we observed:

- The growth curve of $K$. lactis in these conditions is still elevated (maximum absorbance: flask - 1,57 and vial - 2,93), indicating the presence of oxygen;
- A decrease of growth was not observed in the second batch of the cultivation;

- In the previous results with complex medium, growth of $K$. lactis in a sequential batch was lower;

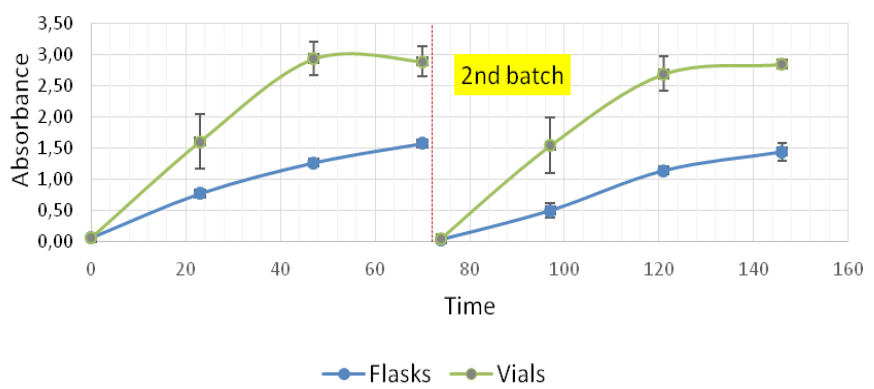

Image 2. Growth of $K$. lactis in syntetic medium without oleate and ergosterol

In the experiment with the presence of the cofactors, the flow of nitrogen used was very low and probably not enough to remove all the oxygen. This could explain the increased growth that was noticed even in the synthetic medium.

\section{Conclusions}

To obtain better results, the experiments in synthetic medium will be repeated with some modifications in the procedure to ensure the anaerobic condition, including the addition of anti-foam.

\section{Acknowledgement}

We acknowledge Fundação de Amparo à Pesquisa do Estado de São Paulo (FAPESP), São Paulo, Brazil, for financial support.

\footnotetext{
Moller, K.; Olsson, L.; Piskur, J.. Journal of bacteriology. 2001, 183, 2485.

${ }^{2}$ Valadi, H. et al. Journal of Microbiological Methods., 2001, 47, 51.
} 